SUPPLEMENT

\title{
Aboriginal users of Canadian quitlines: an exploratory analysis
}

\section{Lynda M Hayward, H Sharon Campbell, Carol Sutherland-Brown}

See end of article for authors' affiliations

Correspondence to Dr Lynda Hayward, Centre for Behavioural Research and Program Evaluation, Lyle S Hallman Institute, Room 1717A, University of Waterloo, 200 University Avenue West, Waterloo, Ontario, Canada, N2L 3G1. lhayward@healthy. uwaterloo.ca

Received 5 January 2007 Accepted 11 July 2007

\begin{abstract}
Objectives: To conduct an exploratory, comparative study of the utilisation and effectiveness of tobacco cessation quitlines among aboriginal and non-aboriginal Canadian smokers.

Setting: Population based quitlines that provide free cessation information, advice and counselling to Canadian smokers.

Subjects: First time quitline callers, age 18 years of age and over, who called the quitline between August 2001 and December 2005 and who completed the evaluation and provided data on their ethnic status $(\mathrm{n}=7082)$.

Main measures: Demographic characteristics and tobacco behaviours of participants at intake and followup; reasons for calling; actions taken toward quitting, and 6-month follow-up quit rates.

Results: $7 \%$ of evaluation participants in the time period reported aboriginal origins. Aboriginal participants were younger than non-aboriginals but had similar smoking status and level of addiction at intake. Concern about future health and current health problems were the most common reasons aboriginal participants called. Six months after intake aboriginals and non-aboriginals had taken similar actions with $57 \%$ making a 24-hour quit attempt. Quit rates were higher for aboriginals than non-aboriginals, particularly for men. The 6-month prolonged abstinence rate for aboriginal men was $16.7 \%$ compared with $7.2 \%$ for aboriginal women and $9.4 \%$ and $8.3 \%$ for non-aboriginal men and women, respectively.

Conclusions: This exploratory analysis showed that even without targeted promotion, aboriginal smokers do call Canadian quitlines, primarily for health related reasons. We also showed that the quitlines are effective at helping them to quit. As a population focused intervention, quitlines can reach a large proportion of smokers in a cost efficient manner. In aboriginal communities where smoking rates exceed $50 \%$ and multiple health risks and chronic diseases already exist, eliminating non-ceremonial tobacco use must be a priority. Our results, although exploratory, suggest quitlines can be an effective addition to aboriginal tobacco cessation strategies.
\end{abstract}

$\mathrm{T}$ he World Health Organization has expressed deep concern about the high tobacco consumption among indigenous peoples and the need to engage these communities in the development and implementation of tobacco control programmes that are culturally appropriate. ${ }^{1}$ The $3.3 \%$ of the Canadian population who identify themselves as aboriginal are culturally and geographically diverse, and can be generally categorised as "North American Indian," "Métis" or "Inuit," with a variety of cultural and/or language groups within these categories. $^{2}$ Estimates of the smoking prevalence among aboriginal Canadians in 2002 ranged from $51.4 \%$ of those living off reservations ${ }^{3}$ to $58.8 \%$ of those living on reservations, ${ }^{4}$ both more than twice the prevalence for the Canadian population in the same year $(21 \%) .^{5}$ This has not changed appreciably since the 1991 Aboriginal Peoples Survey. ${ }^{6}$ Relatively high smoking rates have also been reported for aboriginal populations living in the United States (33\%), ${ }^{7}$ Australia (51\%) and New Zealand (51\%). ${ }^{8}$ Thus an urgent need exists around the world for effective cessation strategies that will engage aboriginal smokers.

Quitlines have developed rapidly over the past 10 years as effective cessation strategies, ${ }^{910}$ yet their effectiveness among aboriginal populations has received little attention to date. In New Zealand, some comparisons have been made between Maori (15\%) and non-Maori callers to the national quitline, examining how they heard about the service, demographic characteristics and the outcomes for those in the nicotine replacement programme offered in conjunction with the quitline counselling. ${ }^{11}$ Callers to the Oklahoma tobacco helpline in the United States have also been surveyed and 7.3\% were found to be American Indians. ${ }^{12}$

In Canada, the renewed Federal Tobacco Control Strategy Program in 2001 specifically allocated funds to address the high smoking rates in First Nations (North American Indian) and Inuit communities with an objective to build capacity within these communities "to develop and deliver comprehensive, culturally sensitive and effective tobacco control programs."13 At that time, there were few examples of culturally appropriate smoking interventions. ${ }^{14}$ Among the tobacco control best practices initially reviewed, telephone support for cessation was viewed as a promising addition, ${ }^{15}$ and the Canadian quitlines were listed as cessation aids in the resource guide developed for aboriginal communities. ${ }^{16}$

In Canada, population based quitlines first began in 2001 in Ontario as part of their provincial tobacco control strategy. By 2003 four other provinces had their own quitline, and five contracted services out. None of the quitlines have eligibility restrictions-all smokers regardless of readiness to quit, insurance status, or previous quit attempts can call the service. Those wishing to help others quit can also call for information on how to support quitters. Callers receive basic information and advice, motivational counselling based on scientific protocols and mailed materials. Proactive services are offered to quitline callers according to their commitment to quit smoking within a given timeframe. Pharmaceutical aids are not

Abbreviations: CBRPE, Centre for Behavioural Research and Program Evaluation; $\mathrm{HSI}$, heaviness of smoking index 
provided. Promotion (mass media, earned media, fax referral, community partnerships) is aimed at all smokers with a primary focus on those ready to quit. To date, Canadian quitlines have not targeted specific populations such as aboriginals.

This paper is an exploratory analysis of the utilisation and effectiveness of Canadian quitlines for smokers with aboriginal ethnic or cultural origins. Two quitlines serving seven provinces (Newfoundland and Labrador, Nova Scotia, Prince Edward Island, New Brunswick, Ontario, Manitoba and Saskatchewan) provided data. We examined caller characteristics (for example, demographics, tobacco behaviours), their reasons for calling and 6-month outcomes (service satisfaction, actions taken towards quitting, and smoking cessation rates). To help us identify unique aspects of aboriginal users, comparisons are made with participants of non-aboriginal ethnic origins.

\section{METHODS}

\section{Quitline evaluation procedure}

Canadian quitlines are evaluated by the Centre for Behavioural Research and Program Evaluation (CBRPE) at the University of Waterloo. At the end of the first client initiated call to the quitline, those over 18 years of age and calling for themselves are asked if they would be willing to participate in the evaluation. The names and contact information of those who agree are sent to CBRPE and callers are subsequently randomised to either a 30-day or 6-month follow-up telephone interview, conducted using WINCATI technology and trained interviewers. Up to 10 call attempts are made to each participant within two weeks of the follow-up date. Those not contacted within the 2 -week period are considered lost to follow-up.

\section{Participants}

Participants were first time callers, age 18 years and over, who called the quitline between August 2001 and December 2005. During this time frame ethnic status was ascertained only at follow-up; hence this paper only includes those who completed an evaluation survey and answered the question on ethnic status.

\section{Data sources and measures}

Data for these analyses originated from two sources: intake data collected by the quitline at the time of the client's first call and data from the follow-up evaluation surveys.

\section{Participant characteristics}

Participant characteristics are reported for all participants in both the 30-day and 6-month evaluations $(n=516)$. The intake data include self reported participant characteristics (sex, age group, education level and smoking status), how they heard about the quitline and the reason for the call noted by the quit specialist/counsellor. The follow-up data include the self reported reason for the initial call. In addition, measures for ethnic origin and tobacco addiction have been created.

Aboriginal status was determined using the Census Canada question that asks respondents to identify the ethnic or cultural origins of their ancestors. ${ }^{17}$ Those who identified themselves as having ancestors of an aboriginal ethnic or cultural origin were classified as aboriginal participants. For the purpose of comparison, those with other ethnic or cultural origins were classified as non-aboriginals.

Level of addiction was measured using the heaviness of smoking index $(\mathrm{HSI})^{18}$ that combines the number of cigarettes smoked per day and the time to first cigarette in the morning. High scores on the HSI indicate higher levels of addiction and greater difficulty in quitting.

\section{Outcome measures}

The outcome measures used in this analysis were collected during the follow-up interview six months after the initial call $(\mathrm{n}=321)$. These include satisfaction with the service (specifically whether they would refer a friend to the service), actions taken towards quitting (including setting a quit date, reduced consumption and making a serious quit attempt by remaining smoke-free for 24 hours), and smoking cessation rates.

Unadjusted 7-day and 30-day point prevalence and 6-month prolonged abstinence rates, based on Society for Research in Nicotine and Tobacco recommendations, ${ }^{19}$ were calculated only for those who completed the follow-up survey. The point prevalence quit rates reflect no smoking, not even a puff, in the previous 7 or 30 days, respectively. The 6-month prolonged abstinence rate defined quitters as not having smoked on seven consecutive days, or more than one day a week during two consecutive weeks, since their call to the quitline.

\section{Data analysis}

The intake and follow-up data were merged and analysed using SAS statistical software, (version 9.1.3). Descriptive statistics were used and comparisons of the aboriginal and nonaboriginal ethnic groups were made using $\chi^{2}$ and Fisher's exact tests.

\section{RESULTS}

Of the 7082 evaluation participants who identified their ethnic origin, $516(7.3 \%)$ stated that they have ancestors of aboriginal ethnic or cultural origin (169 of solely aboriginal origin; 347 with aboriginal origin plus other origins).

\section{Participant characteristics}

Demographic characteristics of aboriginal and non-aboriginal participants who completed either a 30-day or 6-month followup survey are summarised in table 1 . The majority of aboriginal participants are women (69.6\%)-a higher proportion than for non-aboriginal participants $(62.2 \%)$. It is notable that, although men's participation is commonly lower than women's, it is even lower in the aboriginal group. Aboriginal evaluation participants also tend to be younger and have a lower level of education than the non-aboriginal participants.

\section{Tobacco behaviour}

In general, the tobacco behaviours of aboriginal participants are similar to those for participants with non-aboriginal origins, as can be seen in table 2. A large majority (78.1\%) of the aboriginal participants were smokers at intake, as is the case for the non-aboriginal group $(77.5 \%)$. The remaining aboriginal participants had recently quit and were in need of support (21.9\%). Male aboriginal participants were more likely to be current smokers than female aboriginals $(84.7 \%$ vs $75.2 \%$, respectively, $\mathrm{p}=0.016)$, which is also the case for the nonaboriginal group $(79.9 \%$ vs $76.1 \%$, respectively, $p=0.001)$.

As measured by the HSI, aboriginal men also were more addicted to tobacco than aboriginal women $(p=0.001)$. In addition, aboriginal smokers were more likely to live with other people who smoked than smokers in the non-aboriginal group $(\mathrm{p}=0.001)$.

\section{How heard about the quitline}

Aboriginal participants in the quitline evaluation heard about the service from a variety of sources. Referrals were the most common source mentioned by those with aboriginal origins (45.9\% mentioned referrals; $40.1 \%$ of men and $48.5 \%$ of women; $\mathrm{p}=0.085$ ). In particular, aboriginal women were more likely to mention referrals from health professionals than women with non-aboriginal origins (30.9\% and $25.4 \%$, respectively; $\mathrm{p}=0.024$ ). 
Table 1 Demographic characteristics of aboriginal and non-aboriginal participants

\begin{tabular}{lllll}
\hline & Aboriginal & & Non-aboriginal & \\
\cline { 2 - 2 } & No $(\%)$ & No $(\%)$ & p Value \\
\hline Sex & $n=516$ & $n=6566$ & 0.001 \\
$\quad$ Female & $359(69.6)$ & $4087(62.2)$ & \\
Male & $157(30.4)$ & $2479(37.8)$ & \\
Age (years) & $n=500$ & $n=6428$ & 0.000 \\
$18-29$ & $91(18.2)$ & $765(11.9)$ & \\
$30-49$ & $263(52.6)$ & $2973(46.3)$ & \\
$>49$ & $146(29.2)$ & & $2690(41.8)$ & \\
Education level & $n=430$ & $n=5546$ & \\
Less than high school & $128(29.8)$ & $1249(22.5)$ & 0.000 \\
High school & $157(36.5)$ & $1971(35.5)$ & \\
Post-secondary & $145(33.7)$ & $2326(41.9)$ & \\
\hline
\end{tabular}

Aboriginal men were less likely than aboriginal women $(2.6 \%$ vs $9.2 \% ; \mathrm{p}=0.005)$ and men in the non-aboriginal group $(6.9 \%$; $\mathrm{p}=0.031)$ to have referrals from family and friends. However, the aboriginal men were more likely to have referrals from a community organisation than the non-aboriginal men $(8.9 \%$ and $3.8 \%$, respectively; $p=0.005$ ). The most frequently mentioned way aboriginal men heard about the quitline was through media $(42.0 \%)$, predominantly newspapers $(17.8 \%)$ and television $(17.8 \%)$.

\section{Reason for initial call to quitline}

At intake, the quit specialists were asked to note reasons for the initial call. Most evaluation participants with aboriginal origins were interested in help with quitting $(74.7 \%)$, followed by referral to other services $(17.5 \%)$ and general information $(7.0 \%)$. The distribution of these reasons closely resembles that for non-aboriginal participants.

At follow-up, those with aboriginal and non-aboriginal origins also gave similar reasons for calling the quitline. The top three reasons were: concern for their own future health (37.8\% of aboriginals; $46.4 \%$ of non-aboriginals; $p=0.000$ ), current health problems $(29.0 \%$ and $29.6 \%$, respectively; $\mathrm{p}=0.835)$, and the increased cost of smoking (14.6\% and $19.3 \%$ respectively; $p=0.012$ ). Aboriginal participants were less concerned about their own future health and the cost of smoking than those with non-aboriginal origins. Aboriginal men were more likely to mention advice from a healthcare professional than aboriginal women $(11.3 \%$ and $5.5 \%$ respectively; $\mathrm{p}=0.032)$, and increased costs $(21.8 \%$ and $11.5 \%$, respectively; $\mathrm{p}=0.005$ ).

\section{Quitline use}

Use of the service by participants with aboriginal origins is comparable with non-aboriginal participants, with similar proportions making more than one call (14.7\% of aboriginals; $14.9 \%$ of non-aboriginals) and accepting proactive calls from the quit specialists $169.7 \%$ of aboriginals; $68.8 \%$ of nonaboriginals).

\section{Outcome}

Evaluation participants who completed a 6-month follow-up interview provided data for the outcome analysis, which includes satisfaction with the service, and for those who were smoking at the time of intake, actions taken towards quitting and smoking cessation rates.

\section{Satisfaction with the service}

Six months after their initial call, satisfaction with the service was high among both aboriginal and non-aboriginal participants. A large majority of aboriginal participants would recommend the service to a friend $(88.7 \%)$, as is the case for the non-aboriginal group (89.9\%; $p=0.247)$. Satisfaction among aboriginal participants did not differ significantly by sex $(p=0.505)$ or age $(p=0.875)$.

\section{Actions taken towards quitting}

The patterns of action taken towards quitting by participants with aboriginal origins are very similar to those of the nonaboriginal participants. Sizeable proportions of the aboriginal participants in the evaluation had reduced consumption $(73.4 \%)$, set a quit date $(57.4 \%)$ or had stopped for 24 hours $(57.6 \%)$. There was little difference between aboriginal men and women. The use of other smoking cessation programmes and aids by the aboriginal participants in conjunction with the quitline to help them quit tended to be slightly lower but not significantly different from that for the non-aboriginal participants ( $\mathrm{p}$ values $>0.05$ ).

Table 2 Tobacco behaviours of aboriginal and non-aboriginal participants

\begin{tabular}{lllll}
\hline & \multicolumn{2}{c}{ Aboriginal } & & \multicolumn{2}{c}{ Non-aboriginal } & \\
\cline { 2 - 2 } & No $(\%)$ & No $(\%)$ & p Value \\
\hline Smoking status & $\mathrm{n}=516$ & $\mathrm{n}=6568$ & 0.824 \\
$\quad$ Smoker & $403(78.1)$ & $5093(77.5)$ & \\
Recent quitter & $113(21.9)$ & $1471(22.4)$ & \\
Non-smoker & $0(0.0)$ & & $4(0.1)$ & \\
Heaviness of smoking index for smokers & $\mathrm{n}=364$ & $\mathrm{n}=4674$ & 0.784 \\
$\quad$ Low & $97(26.7)$ & $1323(28.3)$ & \\
Medium & $168(46.2)$ & $2126(45.5)$ & \\
High & $99(27.2)$ & $1225(26.2)$ & \\
\hline
\end{tabular}


Table 3 Six-month cessation rates for aboriginal and non-aboriginal smokers

\begin{tabular}{|c|c|c|c|}
\hline & Aboriginal & Non-aboriginal & \\
\hline & No (\%) & No (\%) & p Value \\
\hline 7-day point prevalence & $\begin{array}{l}n=243 \\
46(18.9)\end{array}$ & $\begin{array}{l}n=2953 \\
488(16.5)\end{array}$ & 0.326 \\
\hline Male & $\begin{array}{l}n=90 \\
20(22.2)\end{array}$ & $\begin{array}{l}n=1180 \\
212(18.0)\end{array}$ & 0.322 \\
\hline $\begin{array}{l}\text { Female } \\
\text { (p Value within group) }\end{array}$ & $\begin{array}{l}n=153 \\
26(17.0) \\
(0.315)\end{array}$ & $\begin{array}{l}n=1770 \\
275(15.5) \\
(0.085)\end{array}$ & 0.643 \\
\hline 30-day point prevalence & $\begin{array}{l}n=243 \\
41(16.9)\end{array}$ & $\begin{array}{l}n=2953 \\
419(14.2)\end{array}$ & 0.254 \\
\hline Male & $\begin{array}{l}\mathrm{n}=90 \\
18(20.0)\end{array}$ & $\begin{array}{l}n=1180 \\
181(15.3)\end{array}$ & 0.231 \\
\hline $\begin{array}{l}\text { Female } \\
\text { (p Value within group) }\end{array}$ & $\begin{array}{l}\mathrm{n}=153 \\
23(15.0) \\
(0.376)\end{array}$ & $\begin{array}{l}\mathrm{n}=1770 \\
237(13.4) \\
(0.146)\end{array}$ & 0.539 \\
\hline 6-month prolonged abstinence & $\begin{array}{l}n=243 \\
26(10.7)\end{array}$ & $\begin{array}{l}n=2953 \\
259(8.8)\end{array}$ & 0.293 \\
\hline Male & $\begin{array}{l}\mathrm{n}=90 \\
15(16.7)\end{array}$ & $\begin{array}{l}n=1180 \\
111(9.4)\end{array}$ & 0.041 \\
\hline $\begin{array}{l}\text { Female } \\
\text { (o Value within aroup) }\end{array}$ & $\begin{array}{l}n=153 \\
11(7.2) \\
(0.030)\end{array}$ & $\begin{array}{l}n=1770 \\
147(8.3) \\
10.319)\end{array}$ & 0.759 \\
\hline
\end{tabular}

\section{Smoking cessation rates}

Overall aboriginal cessation rates are comparable to those for the non-aboriginal group (see table 3). However, it is notable that the aboriginal cessation rates tend to be a little higher than for non-aboriginal participants. In particular, aboriginal men have a 6-month prolonged abstinence rate of $16.7 \%$ compared with $9.4 \%$ for non-aboriginal men $(p=0.041)$. Aboriginal men also tend to have higher cessation rates than aboriginal women, particularly for 6-month prolonged abstinence (16.7\% and $7.2 \%$, respectively; $\mathrm{p}=0.030$ ). There is also some indication that young aboriginal adults (18-29 years) tend to have higher 6month prolonged abstinence rates than older aboriginals (14.0\% compared with $7.5 \% ; \mathrm{p}=0.129)$.

\section{DISCUSSION}

This exploratory analysis is one of the few studies to report on aboriginal people's use of tobacco cessation quitline services and to compare utilisation and effectiveness with non-aboriginals. The results suggest that Canadian quitlines are used by aboriginal smokers, even without targeted promotion. Outcomes are similar to those achieved by other smokers, thus suggesting quitlines can be an effective intervention for people of aboriginal origin, particularly men, to help them change their tobacco behaviours.

In Canada, quitlines are operated as part of provincial tobacco control strategies and are population focused interventions. There are no clinical, insurance, smoking related or other such restrictions about who can receive counselling, and protocols are tailored to smokers' readiness to quit, personal smoking history and motivation. The appeal of population based interventions is the ability to reach large numbers of those at risk with an effective intervention in a cost efficient manner. ${ }^{20}$ This is particularly appealing to the community, policy makers and healthcare advocates for reaching aboriginal populations which have tremendous cultural and geographic diversity as in Canada and other countries, particularly the United States. The effectiveness of quitlines in the general population has been well documented. ${ }^{9}{ }^{10}$ Our study suggests quitlines may also be effective for aboriginal populations and that aboriginal peoples will use them.

For any population based intervention to succeed, it must be used by many. Cost efficiencies are achieved by expanding reach (the proportion of the target population calling the quitline $)^{20}$ and effective promotion remains a challenge for most quitlines regardless of the populations served. New Zealand uses promotion strategies targeted specifically to Maoris, ${ }^{11}$ whereas Canadian quitlines currently reach aboriginal smokers without culturally specific promotion. The most common ways aboriginals heard about the quitline was through referrals $(45.9 \%)$ and media $(38 \%)$. Referrals tend to be from community organisations (men) and health professionals (women). Further exploration of the referral potential of community organisations and health professionals currently serving aboriginal populations is recommended.

With regard to service utilisation and subsequent outcomes, the lack of statistically significant differences between the aboriginal and non-aboriginal participants in their utilisation of proactive services or in the actions taken after calling the quitline suggests that the current protocols are equally effective for motivating smokers to quit.

Over half of the aboriginal participants set a quit date (57.4\%) and/or made a 24-hour quit attempt (57.6\%). As noted by $\mathrm{Zhu}^{21}$ quit attempts are a necessary step in ultimately quitting and should be treated as an important indicator of quitline effectiveness.

In this study, unadjusted 6-month prolonged abstinence rates for aboriginal men were high $(16.7 \%)$ as were they for younger aboriginal smokers (14.0\% among 18-30-year-olds). All quit rates were higher for aboriginal men than aboriginal women, even though HSI was higher among the men. We do not have the data to determine why, but there may be cultural factors that differentially influence aboriginal men to call a quitline and to maintain quits compared with aboriginal women.

There are some limitations on the conclusions that can be drawn from this exploratory study. Firstly, our sample size is small and only includes those who completed the evaluation and provided their ethnic status. We cannot assume our findings are representative of either aboriginal quitline callers or the overall aboriginal smoking population in the provinces served by these quitlines. Moreover, the question to determine ethnic status is the one used by Census Canada, to allow comparison to national data. However, anyone with aboriginal 


\section{What this paper adds}

- Vast disparities in non-ceremonial tobacco use exist between aboriginal and non-aboriginal populations with aboriginal smoking rates more than twice those of nonaboriginals. The subsequent disease burden from tobacco use is likewise staggeringly high. To date, most efforts to reduce aboriginal tobacco use have focused on culturally sensitive interventions that are owned and delivered by the community. Unexplored is the potential of cost efficient population based interventions, such as tobacco cessation telephone quitlines, to reach large numbers of aboriginal smokers with an effective intervention. Our exploratory study examined the utilisation and effectiveness of quitlines among Canadian aboriginal smokers.

- Results showed that even without targeted promotion aboriginals are calling the quitlines, largely because of health concerns. They heard about the service through referrals from community organisations (men), health professionals (women) and some media. Such knowledge can help tailor promotion strategies to increase reach, the prime driver of impact at the population level. We also found aboriginal men were significantly more likely to report prolonged abstinence at six months (16.7\%) than aboriginal women $(7.2 \%)$, and non-aboriginals; all of whom received the same cessation intervention. These results are preliminary but raise interesting questions about our approach to the aboriginal smoking epidemic.

ancestors could be included whether or not that person currently considers themselves to be North American Indian, Inuit or Métis. Canadian quitlines have recently implemented the NAQC minimal data set which collects ethnic origins at intake. ${ }^{22}$ This will provide better data for planning and evaluating promotion strategies.

Given that current counselling protocols lead to fairly high quit attempts and prolonged abstinence, our recommendation would be for Canadian quitlines to focus on increasing reach at this time. Other quitlines around the world could also consider this approach. If data on the size of the aboriginal population is not available setting a target for percentage of quitline calls, as in New Zealand, could help direct efforts towards reach. In a population based intervention, where quitlines excel, increased reach has the greatest impact on the overall smoking rate. For geographically dispersed aboriginal populations, where over $50 \%$ of adults smoke daily, this is the only reasonable approach.

\section{ACKNOWLEDGEMENTS}

The authors would like to thank the Canadian quitlines providing services to the provinces of Newfoundland and Labrador, Nova Scotia, Prince Edward Island, New Brunswick, Ontario, Manitoba and Saskatchewan for their collaboration in the study. We would also like to thank the Survey Research Centre at the University of Waterloo for conducting the follow-up evaluation surveys. The Centre for Behavioural Research and Program Evaluation is supported by the National Cancer Institute of Canada with funds from the Canadian Cancer Society. Funding for this study was provided by the Tobacco
Control Programme at Health Canada and the Ontario Division of the Canadian Cancer Society. None of the authors have any affiliation with the tobacco industry.

\section{Authors' affiliations}

Lynda M Hayward, H Sharon Campbell, Centre for Behavioural Research and Program Evaluation, Lyle S Hallman Institute, Room 1717A, University of Waterloo, 200 University Avenue West, Waterloo, ON, Canada, N2L $3 G 1$

Carol Sutherland-Brown, Office of the Minister of Health, Health Canada, 16th Floor Brooke Claxton Building, Address locator 0916A, Ottawa, ON, Canada, K1A OK9

\section{REFERENCES}

1 World Health Organization. WHO framework convention on tobacco control. Geneva: WHO, 2005

2 Statistics Canada. Aboriginal peoples of Canada: a demographic profile. 2002 Census: analysis series. Ottawa, Canada: Minister of Industry, January 2003 (catalogue no 96F0030XIE2001007)).

3 Tiepkema $M$. The health of the off-reserve aboriginal population. Supplement to Health Reports, 2002;13:1-17 (Statistics Canada catalogue 82-003-XIE).

4 First Nations Centre. First Nations Regional Longitudinal Health Survey (RHS) 2002/03. Results for adults, youth and children living in first nations communities. Ottawa, Canada: First Nations Centre, 2005.

5 Health Canada. Smoking in Canada: an overview, Canadian Tobacco Use Monitoring Survey Factsheets.http://www.hc-sc.gc.ca/hl-vs/tobac-tabac/ research-recherche/stat/ctums/fs-if/2002/index_e.html (Accessed 13 December 2006).

6 Millar WJ. Place of birth and ethnic status: factors associated with smoking prevalence among Canadians. Health Reports 1992;4:7-24 (Statistics Canada catalogue 82-003-XIE).

7 Center For Disease Control. Cigarette smoking among adults-United States, 2004. Morb Mort Wkly Rep 2005;54:1121-4.

8 Centre for Excellence in Indigenous Tobacco Control. International comparisons http://www.healthinfonet.ecu.edu.au/ceitc (accessed 19 December 2006).

9 Zhu SH, Anderson CM, Tedeschi GJ, et al. Evidence of real-world effectiveness of a telephone quitline for smokers. N Engl J Med 2002;347:1087-93.

10 Stead LF, Perera R, Lancaster T. A systematic review of interventions for smokers who contact quitlines. Tob Control 2007;16(Suppl I):i3-8.

11 The Quit Group and the Ministry of Health. Maori smoking and tobacco use, Fact Sheet 1. Wellington, New Zealand: Ministry of Health, 2006.

12 Oklahoma Task Force to Eliminate Health Disparities and the Oklahoma Turning Point Council. Tobacco-Related Disparities in Oklahoma. Newsletter, 2005;1:1-4.http://www.health.state.ok.us/COMMISH/hd/ Tobacco\%20newsletter.pdf (accessed 7 December 2006).

13 First Nations and Invit Health Branch and the First Nations and Inuit Tobacco Control Strategy Advisory Circle. First Nations \& Invit tobacco control strategy program framework, August 2002:4.http://www.hc-sc.gc.ca/fnih-spni/pubs/ tobac-tabac/2002_frame-cadre/index_e.html (accessed 20 December 2006).

14 Tobacco Control Programme, Health Canada. Tobacco use cessation programs and resources: 2000 update. Ottawa, Canada: Minister of Supply and Services, 2000.

15 Marriott J, Mable AL. Aboriginal tobacco control: promising strategies and potential for best practices, report prepared for the First Nations and Invit Tobacco Control Strategy, Health Canada First Nations and Invit Health Branch and the National Aboriginal Health Organization, 2002.

16 Boomer J. Building and sustaining partnerships: a resource guide to address non-traditional tobacco use, prepared for First Nations and Invit Health Branch, Health Canada, October 2003. http://www.hc-sc.gc.ca/fnih-spni/pubs/tobactabac/2003_sust-maint_part/index_e.html (accessed December 20, 2006).

17 Statistics Canada. Census questionnaires. 2001. http://www12.statcan.ca/ english/census01/home/questionnaire.cfm (accessed 21 December 2006).

18 Heatherton T, Kozlowski L, Frecker R, et al. The Flagerström test for nicotine dependence: a revision of the Flagerström Tolerance Questionnaire. Br J Addict 1991;86:1119-27.

19 Hughes JR, Keely JP, Niaura RS, et al. Measures of abstinence in clinical trials: issues and recommendations. Nicotine Tob Res 2003;6:863-4.

20 Glasgow RE, Vogt TM, Boles SM. Evaluation the public health impact of health promotion interventions: the RE-AIM framework. Am J Public Health 1999b;89:1322-7.

21 Zhu SH. Increasing cessation in the population: quit attempts vs. successful quit attempts. Presented at the World Conference on Tobacco OR Health. Washington, DC, 12-15 July, 2006.

22 Campbell HS, Ossip-Klein D, Bailey L, et al. Minimal dataset for quitlines: a best practice. Tob Control 2007; 16(Suppl I):i16-20 